Research Article

\title{
Measurement of Holistic Road Damage and Analysis of Its Corresponding Traffic Characteristics
}

\author{
Shun Guo $\mathbb{D}^{1,2,3}$ Zhengfeng Huang $\mathbb{D}^{1,2,3}$ Pengjun Zheng, ${ }^{1,2,3}$ Ting Lu $\mathbb{D}^{1,2,3}$ Lili Lu, ${ }^{1,2,3}$ \\ and Xuan Li $\mathbf{i}^{1,2,3}$ \\ ${ }^{1}$ Faculty of Maritime and Transportation, Ningbo University, Ningbo 574, China \\ ${ }^{2}$ Jiangsu Province Collaborative Innovation Center for Modern Urban Traffic Technologies, Nanjing 25, China \\ ${ }^{3}$ National Traffic Management Engineering \& Technology Research Centre Ningbo University Sub-centre, Ningbo 574, China
}

Correspondence should be addressed to Zhengfeng Huang; huangzhengfeng@nbu.edu.cn

Received 10 December 2018; Revised 1 April 2019; Accepted 5 May 2019; Published 25 June 2019

Academic Editor: Eul-Bum Lee

Copyright (C) 2019 Shun Guo et al. This is an open access article distributed under the Creative Commons Attribution License, which permits unrestricted use, distribution, and reproduction in any medium, provided the original work is properly cited.

Many pavement distress (PD) indexes are defined only from the perspective of the PD physical structure, which cannot describe the change of traffic efficiency. Some PD indexes are defined considering the influence of traffic efficiency, but it is unpractical to calculate them out due to their complex processes. We quantify the impact of the cohesive whole of PD points on the traffic flow and also define the corresponding PD index which is easy to compute. Firstly, the lane cell based on the idea of road discretization is introduced. Secondly, the PD degree for the lane cell is defined by the reduction of the average vehicle speed given the same flow condition. The cell whose PD degree is larger than 0 is defined as the PD cell. Then, considering (1) the ratio of PD cells, (2) PD cell distribution, and (3) the influence of the vehicle lane changing, we define the holistic road-damage degree (HRDD) as the PD index. At last, the relationship among HRDD, flow speed, and flow volume is analyzed through the simulated experiments. The results show that (1) the average speed is inversely proportional to HRDD, and the reduction of vehicle speed is more significant with the increase of the traffic flow input; (2) the inverse relation between road capacity and HRDD can be seen on the whole HRDD range. In another word, the proposed HRDD describes the change of traffic efficiency indeed.

\section{Introduction}

Due to the impact of road-use age, vehicle overload, road construction, and bad weather, there are lots of roads in bad conditions in many large- and medium-sized cities. Specifically, many roads connecting mining areas or ports to cities are broken. Pavement distress (PD) has negative effects (vehicle delays, congestion, and overflow) on urban traffic flow. It greatly affects urban commuting travel. So, how to quantify the PD degree accurately is of great significance for urban traffic organization and management.

Since the 1960s, studies on evaluation of the PD degree are divided into two categories. The first type could be found in some industry standards, such as the Present Serviceability Index [1] and the Pavement Condition Index (PCI) model [2] in America, the Maintenance Control Index model [3] in Japan, the Road Condition Indicator model [4] in the UK, and the Pavement Quality and Performance Index model [5] in China. These indexes only focus on the physical structural factors, such as the International Roughness Index (IRI) of the road, road surface roughness [6], road rutting depth, road structure strength, and road lateral force. These studies do not consider the characterization of traffic efficiency.

The second type of study is about the correlation between the PD degree and traffic efficiency. It is divided into two branches. One branch mainly focuses on the sensitivity of traffic efficiency to the PD degree. The results show that the same PD could have different influences on varied weight vehicles in terms of speed sensitivity [7]. The increase of the PD degree could cause an obvious decline of road capacity [8] and rising of the travel time [9]. The other branch is to establish the correlation index of the traffic efficiency to the PD degree. The indicators could reflect the traffic efficiency, 
such as trapezoidal flow rate contractions (TFC) and road impact factor (RIF). TFC [10] is the ratio of difference in traffic flow to density. The two densities correspond to the maximum flow scenarios of good road and certain PD road separately. It could show the reduction degree of road capacity. However, its acquisition requires drawing a complete flow-density curve, which need collecting large amount of data in different flow scenarios. RIF [11] is calculated by the changes of speed and acceleration of many test vehicles in the road surface. These changes are in the horizontal and vertical directions on the condition of nonuniform speed. But its cost is higher because it relies on a huge number of experimental facilities.

On the whole, most current PD indicators only consider the physical structure characteristics of broken road and cannot reflect the magnitude of traffic efficiency. Few PD indexes take traffic efficiency parameters into account, but the calculation process of these factors is complex and collecting data is time-consuming. Despite the aforementioned shortcomings, one thing is certain: traffic efficiency is related to road damage and corresponding vehicle lane-changing behavior. These factors include the PD point features (PD degree, scale, and distribution) and the ratio of vehicle lane changing. Their influences on traffic efficiency could be described as follows. If PD points are taken as the crosswalks, the effect of pedestrian crossing time, crosswalk number, and crosswalk spacing on the traffic efficiency is the well-known phenomenon $[12,13]$. If PD points are taken as the bus stops, traffic efficiency is influenced by lane changing of private vehicles due to the temporary parking of the reaching bus $[14,15]$.

This study proposes the holistic road-damage degree (HRDD) index by considering the relation to traffic efficiency, the easiness of application, the data availability of subparameters, and the physical structure of broken road. This point is our main innovation. In order to demonstrate the feasibility of the HRDD index, we adopt traffic simulation and flow characteristic analysis. So, HRDD definition and its feasibility study is the main content in this paper. The paper is structured as follows. Firstly, the road is divided into lane cells, and the PD degree for lane cell is quantized. Secondly, the density coefficient $\left(f_{1}\right)$ of PD cells is defined to describe the scale of $\mathrm{PD}$ points; the dispersion coefficient $\left(f_{2}\right)$ of PD cells is defined to describe the distribution of PD points; the lane-changing coefficient $\left(f_{3}\right)$ is defined to describe the disorder caused by lane changing in PD cells; and then the HRDD is defined with these coefficients comprehensively. In the end, the simulation experiments of different scenarios of multidamage points are designed. On the one hand, it is used to expound the traffic characteristics with the changing of HRDD. On the other hand, it is used to analyze the reasonability of the impact of HRDD on flow speed and road capacity. The HRDD index can be used as a reference for traffic impact analysis on the PD road.

\section{PD Degree of the Lane Cell}

2.1. Design of Lane-Cell Length. The locations and the magnitude of the pothole are random in real life. In order to analyze the PD magnitude of the holistic road, the road lanes are divided into many lane cells firstly and then the lane cells are aggregated to obtain the HRDD. Assuming that the length of the research segment is $l$ and the length of a lane cell is $l_{0}$, the number of lane cells in one road lane is calculated as $m=\left|l / l_{0}\right|$. If there are $n$ lanes on the road, then the research segment has $m \times n$ lane cells, as shown in Figure 1(a).

Length $l_{0}$ of the lane cell is calculated based on the heaviest pothole, and its constituents are shown in Figure 1(b). Assume that the pothole is located in the center of the lane cell. The length $l_{1}$ of the low-speed zone is assigned with the length of the heaviest pothole from the historical data. The length $l_{2}$ of the buffer zone is acquired by considering the average speed in the low-speed zone, freeflow speed, and the accelerationldeceleration speed of HGVs. So, the length $l_{0}$ is calculated as follows:

$$
\begin{aligned}
& l_{0}=l_{1}+2 \times l_{2}, \\
& l_{2}=\frac{\left(v_{0}^{2}-v_{h}^{2}\right)}{2 a},
\end{aligned}
$$

where $v_{h}$ is the average speed of HGVs in the low-speed zone of heaviest pothole scenario, $v_{0}$ is the free-flow speed of HGVs, $a$ is the average acceleration of HGVs in the buffer zone. As for the general PD cell (as shown in Figure 1(c)), its constituents are different with the heaviest pothole scenario. The speed in the low-speed zone (its length is $\left.l_{1}^{\prime}\right)$ is $v_{g}\left(v_{g} \geq v_{h}\right)$. The length $l_{2}^{\prime}$ in the buffer zone is influenced by the same factors in the heaviest pothole scenario. The difference is that there is a length $l_{3}$ in the free-flow zone in both ends of the lane cell. Vehicles could run in free-flow speed in this zone.

2.2. Calculation of the PD Degree of the Lane Cell. The PD degree $\gamma_{i}$ is defined to describe the reduction of traffic efficiency taking maximum speed as reference in lane cell $i$. The calculation of $\gamma_{i}$ is based on the average reduction ratio of HGV speed under free-flow conditions. It is related to (1) the currently average speed $\bar{v}_{i}$ in the lane cell $i$, (2) the minimum speed $\bar{v}_{\text {min }}$ in the heaviest PD scenario, and (3) the maximum speed $\bar{v}_{\max }$ in lane cell without PD. It is calculated via equation (2). The result belongs to the discrete set $\{0$, $0.25,0.5,0.75,1\}$. The larger the value is, the more serious the $\mathrm{PD}$ is. When $\gamma_{i}=0$, it means that there is no PD in lane cell $i$. On the contrary, $\gamma_{i}>0$ means that lane cell $i$ is a PD cell:

$$
\gamma_{i}=\frac{\left|\left(\left(\bar{v}_{\text {max }}-\bar{v}_{i}\right) /\left(\bar{v}_{\text {max }}-\bar{v}_{\text {min }}\right)\right) \times 4\right|}{4} .
$$

The three speed indexes in equation (2) are calculated as follows:

$$
\begin{aligned}
\bar{v}_{\max } & =v_{0}, \\
\bar{v}_{\min } & =\frac{l_{0}}{2\left(v_{0}-v_{h}\right) / a+l_{1} / v_{h}}, \\
\bar{v}_{i} & =\frac{l_{0}}{2\left(v_{0}-v_{g}\right) / a+l_{1}^{\prime} / v_{g}+2\left\{l_{0}-l_{1}^{\prime}-\left[\left(v_{0}^{2}-v_{g}^{2}\right) / a\right]\right\} / v_{0}} .
\end{aligned}
$$




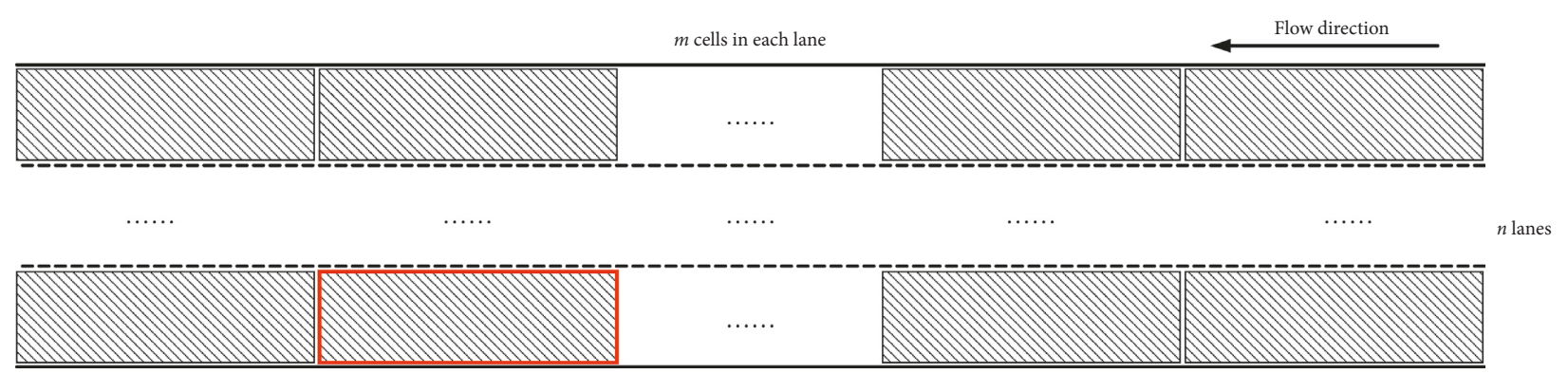

(a)

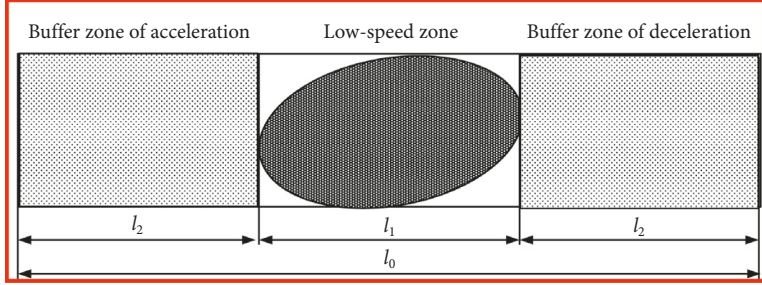

(b)

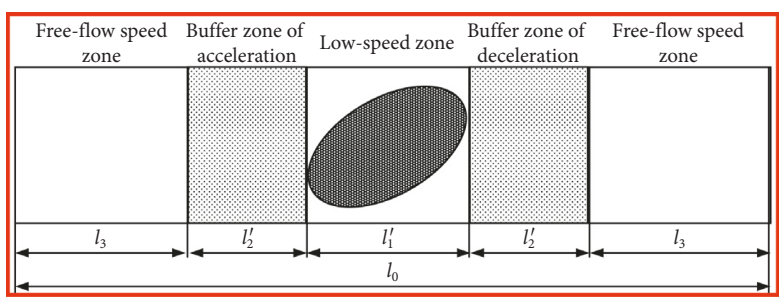

(c)

FIGURE 1: Lane-cell division on the research segment and the constituents of the PD cell. (a) Lane-cell division on the investigated segment. (b) The heaviest PD cell. (c) The general PD cell.

\section{HRDD}

3.1. Definition and Calculation. HRDD describes the impact of all PD cells on traffic efficiency. It is related to various factors such as (1) the PD degree of each lane cell, (2) the research segment length, (3) the number of lanes, (4) the density and dispersion of PD cells, and (5) the lane-changing behavior. HRDD is designed as the multiplication of the aggregated $\mathrm{PD}$ value and the weighted sum of relevant factors, which is shown in the following equation:

$$
\mathrm{HRDD}=D_{0}\left[\omega_{1} f_{1}+\omega_{2} f_{2}+\left(1-\omega_{1}-\omega_{2}\right) f_{3}\right]
$$

where $D_{0}$ is the aggregated PD value of research segment; $f_{1}$ is the density coefficient of PD cells; $f_{2}$ is the dispersion coefficient of PD cells; $f_{3}$ is the lane-changing coefficient; and $\omega_{1}, \omega_{2}$, and $1-\omega_{1}-\omega_{2}$ are the weighted values of $f_{1}, f_{2}$, and $f_{3}$, respectively. The relationship among $\omega_{1}, \omega_{2}$, and $1-\omega_{1}-\omega_{2}$ is $\omega_{1}>\omega_{2}>1-\omega_{1}-\omega_{2}$ according to the significance order of each factor. In the subsequent case study, the weights of $f_{1}, f_{2}$, and $f_{3}$ are $0.7,0.2$, and 0.1 , respectively.

3.2. Aggregated PD Value of the Research Segment. The aggregated PD value $D_{0}$ of the research segment reflects the relative PD magnitude of all PD cells. It is calculated via the Sigmoid function:

$$
D_{0}=\frac{1}{1+e^{-\mu\left(\sum_{j \in \Omega} \gamma_{j}-(N / 2)\right)}},
$$

where $N$ is the number of PD cells, $\Omega$ is the aggregation of $\mathrm{PD}$ cells, and $\mu$ is a tilt-adjusting constant for the Sigmoid function. In the following case study, $\mu=0.63$. This assignment keeps $D_{0}$ being uniformly dispersed when PD scenarios are generated at random in case study.
3.3. Density Coefficient of PD Cells. The density coefficient $f_{1}$ of PD cells is used to describe the PD cell proportion on the whole road. The combination patterns of varied PD cells are shown in Figure 2.

The shaded rectangles represent the PD cells. When the number of PD cells is increasing, the times of vehicle deceleration would rise and the impact on traffic efficiency would enlarge. Therefore, the value of $f_{1}$ has a positive relationship with the proportion of PD cells. The more the PD cells are, the higher the $f_{1}$ is. $f_{1}$ is calculated as follows:

$$
f_{1}= \begin{cases}0, & 0.00 \leq c \leq 0.20 \\ 0.2, & 0.20<c \leq 0.25 \\ 0.4, & 0.25<c \leq 0.35 \\ 0.6, & 0.35<c \leq 0.50 \\ 0.8, & 0.50<c \leq 0.70 \\ 1, & 0.70<c \leq 1.00\end{cases}
$$

where $c$ is the proportion of PD cells and its formula is shown in the following equation:

$$
c=\frac{N}{m \times n} \text {. }
$$

3.4. Dispersion Coefficient of PD Cells. Coefficient $f_{2}$ describes the distribution magnitude of PD cells on the research segment. The more dispersed the PD cells are, the further the traffic efficiency drops are. The reason is that decentralized PD cells could cause frequent vehicle deceleration. So, the value of $f_{2}$ is higher when the PD cells are in a more dispersed situation. The evaluation of $f_{2}$ is mainly based on the average distance $u$ among all centers of PD cells. $u$ is calculated as follows: 


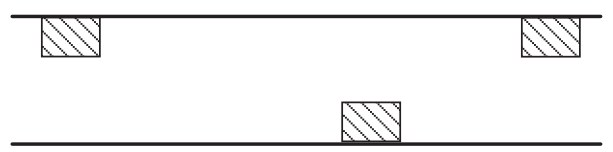

(a)

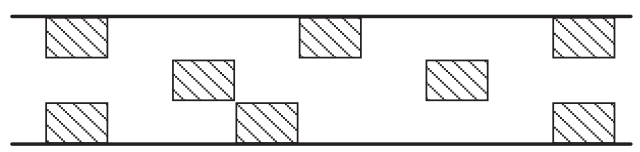

(b)

FIgURE 2: Comparison between PD scenarios of high and low densities: (a) $f_{1}$ is small; (b) $f_{1}$ is large.

$$
u=\frac{\sum_{j \in \Omega}\left|x_{j}-\sum_{j \in \Omega}\left(\left(x_{j} \cdot \gamma_{j}\right) / \sum_{j \in \Omega} \gamma_{j}\right)\right|}{N},
$$

where $x_{j}$ is the center position value of PD cell $j$ from the origin of the research segment when taking the road line as the axis. Finally, $f_{2}$ is calculated by inputting $u$ into the following equation:

$$
f_{2}= \begin{cases}0, & 0 \leq u \leq 0.05 l \\ 0.2, & 0.05 l<u \leq 0.1 l \\ 0.4, & 0.1 l<u \leq 0.15 l \\ 0.6, & 0.15 l<u \leq 0.2 l \\ 0.8, & 0.2 l<u<0.25 l \\ 1, & u \geq 0.25 l\end{cases}
$$

The distribution of 9 PD cells is gradually dispersed, and $f_{2}$ is gradually increased, as shown in subgraphs $(a) \sim(f)$ of Figure 3. This trend is consistent with the real situation: as the subgraphs $(a)$ and $(b)$ show, vehicles only decelerate once; however, in subgraphs $(e)$ and $(f)$, vehicles need to decelerate three times, consequently resulting in greater reduction in traffic efficiency.

3.5. Lane-Changing Coefficient. Historical data show that the proportion of lane-changing vehicles behind varied potholes ranges from 0 to $80 \%$. The higher the proportion is, the more chaotic the traffic flow is, and the lower the travel efficiency is. Herein, $f_{3}$ is defined to describe the chaotic degree of the flow. It is calculated as follows:

$$
f_{3}=-p^{\Sigma_{\gamma_{r}>\gamma_{s}} \lambda_{r s} / M}+1 \text {, }
$$

where $\lambda_{r s}$ is the probability of the vehicle changing into the neighbouring lane cells from its own lane cell $r$ under the condition $\gamma_{r}>\gamma_{s}$ (vehicles change lane from the worse lane into a better lane). $M$ is the total scenario number that satisfies the above condition. $p$ is a parameter between 0 and 1 (in this case study, $p$ is 0.15 ). The lane-changing ratio $\lambda_{r s}$ of each vehicle type is affected by the $\mathrm{PD}$ degree of lane cell, the safe lane-changing spacing, the gap acceptance on neighbouring lane, and so on. Considering these factors, the formula of $\lambda_{r s}$ is established based on the binary logit model $[16,17]$, as shown in the following equation:

$$
\lambda_{r s}=\frac{\exp \left(\alpha_{1} \gamma_{s}+\alpha_{2} T+\alpha_{3} q\right)}{\left\{\exp \left(\alpha_{1} \gamma_{r}+\alpha_{4}\right)+\exp \left(\alpha_{1} \gamma_{s}+\alpha_{2} T+\alpha_{3} q\right)\right\}}, \quad \forall \gamma_{r}>\gamma_{s},
$$

where $T$ is the code of the vehicle type (HGV 1 , car 0 ), reflecting the dummy variable of the safe lane-changing spacing; $q$ (the average flow per hour in one lane) reflects the dummy variable of the gap acceptance degree influenced by the traffic flow $Q$. When $Q<100 \mathrm{veh} / \mathrm{h}, 100-300 \mathrm{veh} / \mathrm{h}$, 300-600 veh/h, 600-900 veh/h, 900-1200 veh/h, and $>1200 \mathrm{veh} / \mathrm{h}$, the corresponding values of $q$ are 5, 4, 3, 2, 1, and 0 , respectively. Even at a fixed $Q$, the variation in traffic density is still large in a short period of time. However, from a macroperspective, the magnitude of traffic density is directly related to the magnitude of $Q$. So, the magnitude of $Q$ is used to characterize the magnitude of $q$ in this paper.

It should be noted that this model applies to the static lane-changing ratio of the scenario. The parameters are calibrated by the inputs of (1) the PD degree of lane cell, (2) the vehicle type, and (3) the traffic flow input. The fitting results are as shown in Table 1 .

The least absolute value of $t$ test for each coefficient is 2.18 (confidence level is 95\%). It is higher than 1.96. It means that all these variables have an obvious influence on the lanechanging behavior. The McFadden determination coefficient $\rho^{2}$ is used to judge fitting goodness of model. When $\rho^{2}$ is between 0.2 and 0.4 , it means that the fitting goodness performs well. In this paper, the $\rho^{2}$ of the lane-changing model is 0.34 , and thus the model is consistent with the actual situation.

\section{Design of Simulation Experiments}

It is hard to find all the scenarios of multi-PD cell combinations in actual life, so the simulation experiments are designed to increase data samples. Assuming that the research segment is divided into 40 cells, there are $5^{40} \mathrm{PD}$ scenarios (40 lane cells with 5 PD degrees) totally by enumerating. In order to ensure the relative uniformity of the sample distribution and reduce the workload simultaneously, the following stratified random sampling method is adapted to design the experiments.

Step 1. The HRDD is uniformly divided into 10 intervals. The upper limit of experiment scenarios in each interval is set to 10 . The initial value of experiment scenarios in each interval is set as $D_{k}=0, k=1,2, \ldots, h, \ldots, 10$.

Step 2. The assumption of uniformly distributed PD degree for each cell is made. $40 \mathrm{PD}$ values (each cell corresponding to a PD value) are extracted independently to generate a set of inputs for an experiment. Judge the subinterval $h$ where its HRDD falls.

Step 3. If $D_{h}=10$, then let $h=h+1$ and then return to Step 2 . Otherwise, let $D_{h}=D_{h}+1$ and add this experiment to the simulation group. 


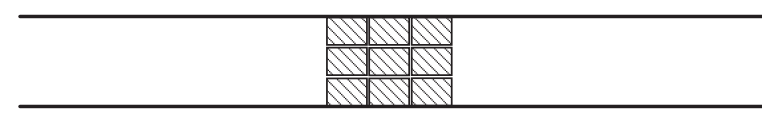

(a)

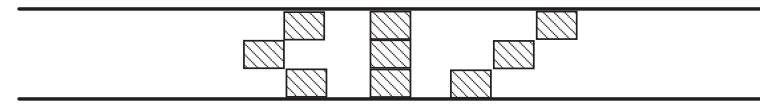

(c)

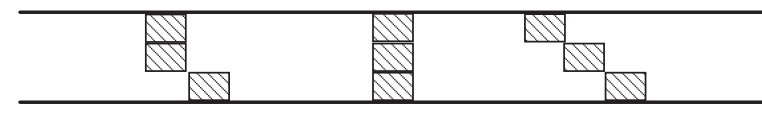

(e)

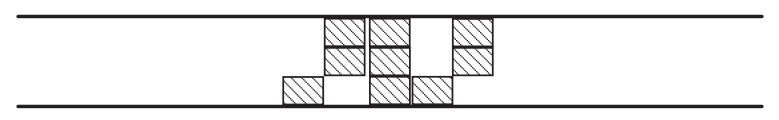

(b)

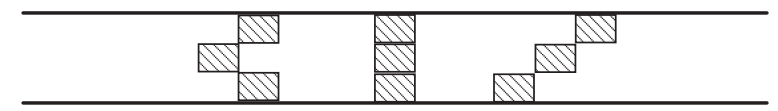

(d)

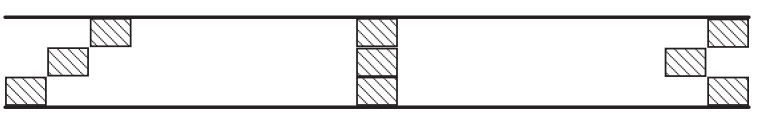

(f)

Figure 3: PD cell distributions corresponding to different values of $f_{2}$ : (a) $u=0.047 l, f_{2}=0$; (b) $u=0.056 l, f_{2}=0.2 ;$ (c) $u=0.138 l, f_{2}=0.4$; (d) $u=0.167 l, f_{2}=0.6$; (e) $u=0.226 l, f_{2}=0.8$; (f) $u=0.382 l, f_{2}=1.0$.

TABLE 1: Fitting results of the binary logit model.

\begin{tabular}{lcccc}
\hline Coefficient & $\alpha_{1}$ & $\alpha_{2}$ & $\alpha_{3}$ & $\alpha_{4}$ \\
\hline Value & -1.96 & -0.16 & 1.04 & 4.29 \\
$T$ test & -2.18 & -4.326 & 3.18 & 3.37 \\
\hline
\end{tabular}

Step 4. If $\sum_{k=1}^{10} D_{k}<100$, then return to Step 2 .

Step 5. If the 100 experiment scenarios extracted satisfy the condition that $D_{0}, f_{1}, f_{2}$, and $f_{3}$ are relatively dispersed (at least one number per 0.1 interval exists for each coefficient), it means that the sampling is successful. Otherwise, half of the samples are rejected randomly and returned to Step 2.

\section{Case Study}

5.1. Description of the Research Segment. A segment of Fenghua Road in Ningbo City was selected. Fenghua Road is an artery undertaking both commuting and freight transport. There is a high ratio of HGVs in its traffic components. Because of the early construction of Fenghua Road, PD point occurs frequently. One bad surface condition is shown in Figure 4.

During the road maintenance from November 2015 to the end of August 2018, Fenghua Road was narrowed into two-way four lanes (previously two-way six lanes). The starting point of the research segment is the South Gate of Ningbo University of Technology. The distance between the starting point and the westbound ending point is about $1 \mathrm{~km}$. The traffic survey was organized in September 2017. During that time, the potholes of the research segment were mainly located at I, II, III, and IV, as shown in Figure 5.

The private cars, HGVs, and buses accounted for $52.7 \%$, $42.6 \%$, and $4.7 \%$ respectively, in this segment. When no pothole occurs on this road, the free-flow speed of private cars, HGVs, and buses are $50 \mathrm{~km} / \mathrm{h}, 40 \mathrm{~km} / \mathrm{h}$, and $40 \mathrm{~km} / \mathrm{h}$, respectively. The arrival flow in the rush hour is about $1700-1900 \mathrm{veh} / \mathrm{h}$, and the flow rate in the off-peak period is about $1200-1400 \mathrm{veh} / \mathrm{h}$ during the survey period. The time distribution of the upstream arrival flow of the day is shown in Figure 6.
5.2. Determination of the Lane-Cell Length. The lane-cell length is determined by the heaviest pothole on the whole Fenghua Road during the construction and reparation period. The relevant parameters obtained by the survey are shown in Table 2.

According to equation (1), the length $l_{2}$ of the buffer zone is $18.35 \mathrm{~m}$. Thus, the length $l_{0}$ of the lane cell is $49.56 \mathrm{~m}$. $l_{0}$ is set to $50 \mathrm{~m}$ by rounding. Thereby, the investigated link is divided into 40 lane cells.

5.3. Calculation of HRDD. Take the center position of the lane cell as the $x$-axis and the corresponding vehicle speed as the $y$-axis. The speed characteristics of different positions are described by the maximum speed $v_{\max }$, the 85 th percentile speed $v_{85}$, the average speed $\bar{v}_{\mathrm{s}}$, the 15 th percentile speed $v_{15}$, and the minimum speed $v_{\min }$, as shown in Figure 7 . The segment can be divided into three areas based on pothole positions and speed classification: upstream road of pothole I $(x<225 \mathrm{~m})$, road between two potholes I and III $(225<x<675 \mathrm{~m})$, and downstream road of pothole III $(x>675 \mathrm{~m})$. Regarding the trend of $\bar{v}_{s}$, it descends with the increase of cell code in the first area; it rises first and then descends in the second area; it rises to approximate the normal speed and then maintains the speed till out of the segment. The speed trend is in a $W$-shape throughout the entire segment. From the perspective of speed stability, the speed dispersion $\left(v_{85}-v_{15}\right)$ is large in the first area; it diffuses rapidly first and then aggregates in the second area, and it aggregates rapidly in the third area.

In Figure 7, the speed of each section reflects the average value of two side-by-side lane cells. Actually, we also recorded the speed of each lane cell (specifically for positions I, II, III, and IV). Provided vehicle speed of the lane cell, the PD degrees of lane cell I, II, III, and IV are $0.5,0.25,0.25$, and 1 , respectively, via calculation of equation (2).

In this PD scenario, $D_{0}=0.5, f_{1}=0, f_{2}=0.8$, and $f_{3}=0.35$. Therefore, the HRDD of this scenario is 0.1 .

5.4. Simulation Settings. In this paper, the lane cell is set by the reduced speed areas that the average speed of the reduced speed area is equal to the average speed of passing the 


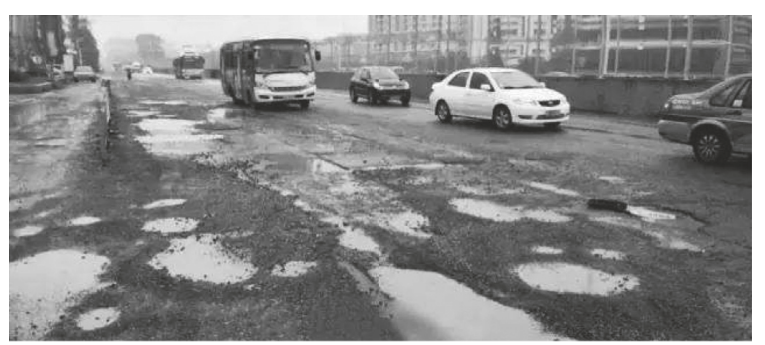

(a)

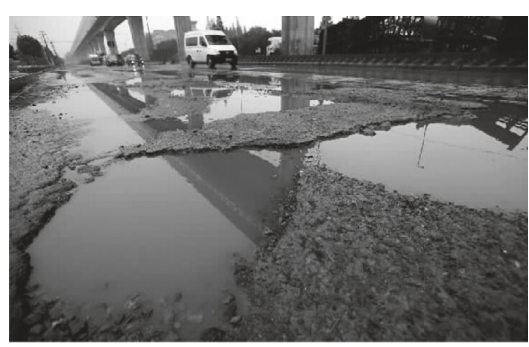

(b)

Figure 4: Bad surface condition on Fenghua Road.

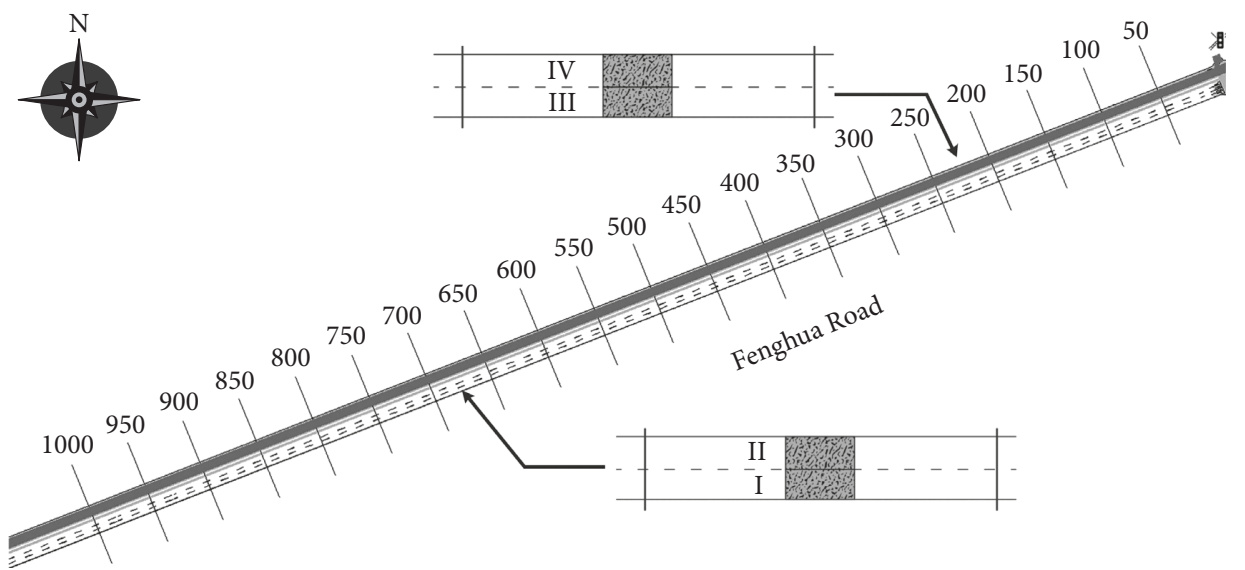

Figure 5: Distribution of potholes on Fenghua Road.

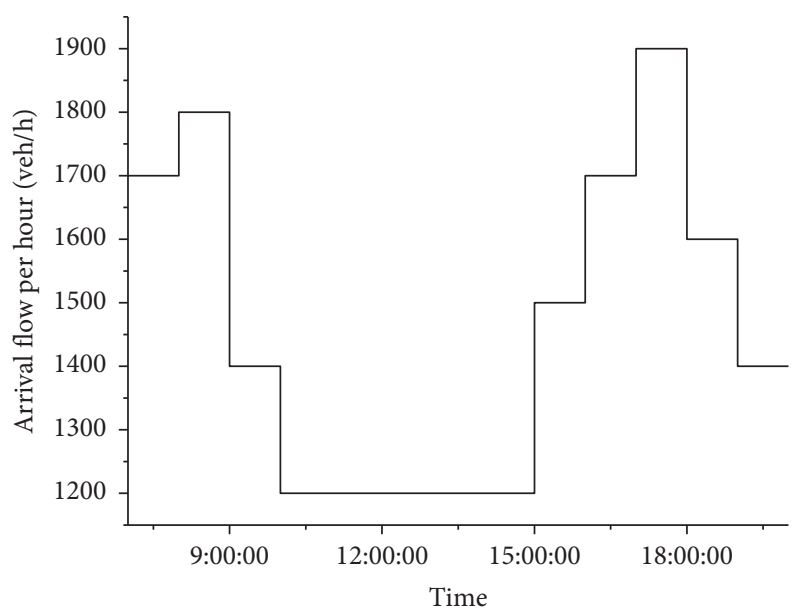

FIgURE 6: Dynamic distribution of the upstream arrival flow on Fenghua Road.

TABLE 2: Traffic characteristic parameters of the heaviest pothole.

\begin{tabular}{lccccccc}
\hline Parameter & \multicolumn{1}{c}{$l_{1}$} & HGV & $v_{h}$ & Car & HGV & Car & HGV \\
\hline Value & $12.86 \mathrm{~m}$ & $2.22 \mathrm{~m} / \mathrm{s}$ & $3.12 \mathrm{~m} / \mathrm{s}$ & $12.8 \mathrm{~m} / \mathrm{s}$ & $13.8 \mathrm{~m} / \mathrm{s}$ & $3.33 \mathrm{~m} / \mathrm{s}^{2}$ & $4.46 \mathrm{~m} / \mathrm{s}^{2}$ \\
\hline
\end{tabular}

corresponding PD cell. In other words, it is necessary to convert $\gamma_{i}$ into desired speed in the lane cell. The matching results between $\gamma_{i}$ and the desired speed are shown in
Table 3. Among them, the upper and lower bounds of the desired speed correspond to the maximal and minimal speeds in equation (2). 


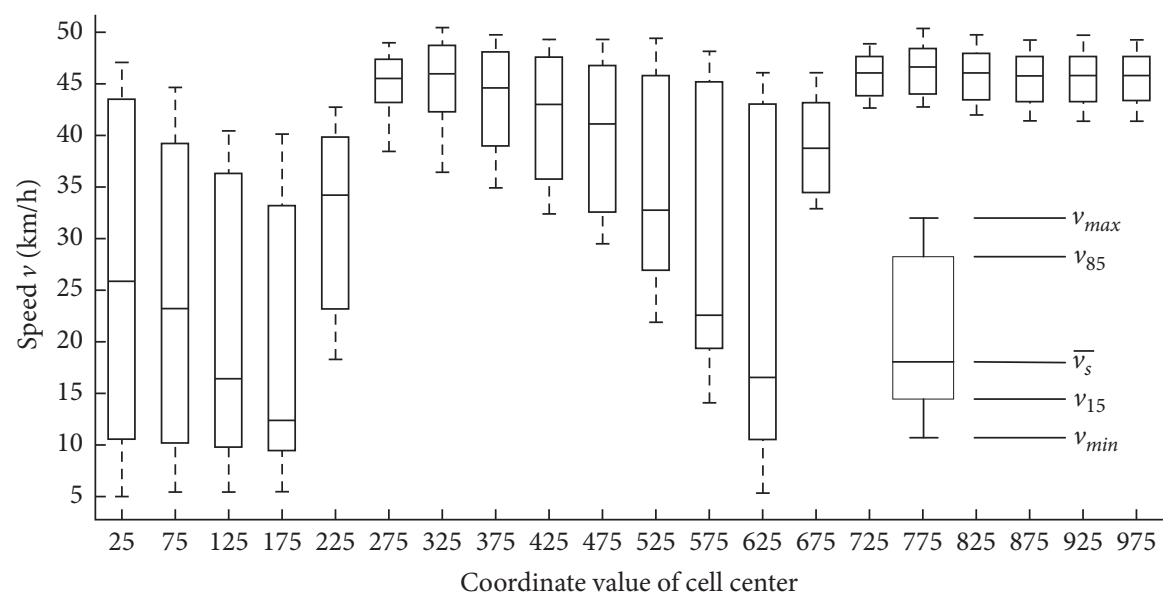

FIgURE 7: Speed characteristics of different positions on the investigated section.

TABLE 3: Matching results between the PD degree and the desired speed.

\begin{tabular}{lcc}
\hline$\gamma_{i}$ & \multicolumn{2}{c}{ Desired speed $(\mathrm{km} / \mathrm{h})$} \\
& Car & HGV and bus \\
\hline 0.00 & $44 \sim 58$ & $40 \sim 48$ \\
0.25 & $34 \sim 44$ & $32 \sim 40$ \\
0.50 & $26 \sim 34$ & $24 \sim 32$ \\
0.75 & $18 \sim 26$ & $16 \sim 24$ \\
1.00 & $10 \sim 18$ & $5 \sim 16$ \\
\hline
\end{tabular}

5.5. Scenario Design of PD Point Combination. To study the degree influence of PD on traffic efficiency in different flow periods, the range of flow input in simulation is 400 2000 with the interval of 100 . The stratified sampling method was used to design $100 \mathrm{PD}$ point scenarios for traffic characteristic analysis. In order to check whether the values of the coefficients tour each subinterval within their ranges, the values of $D_{0}, f_{1}, f_{2}$, and $f_{3}$ are taken as the $x$-axis with an interval of 0.1 and the counts of occurrence are taken as the $y$-axis. The frequency histograms of each coefficient are shown in Figure 8 . The results show that $D_{0}, f_{1}, f_{2}$, and $f_{3}$ of the 100 simulation scenarios generated could tour all the subintervals.

\subsection{Analysis of Traffic Characteristics}

5.6.1. Overall Speed $\bar{v}$ on the Segment. HRDD is taken as the $x$-axis, and the overall speed $\bar{v}$ on the segment is taken as the $y$-axis. The relationship between HRDD and $\bar{v}$ under different flow inputs is plotted as shown in Figure 9.

Figure 9 shows the tendency that the $\bar{v}$ on the whole research segment decreases with the increase of HRDD, which demonstrate the effective relation between the proposed HRDD and traffic efficiency. The absolute value of slope of $\bar{v}$ is 26.27 45.22 under the low-level range of HRDD $(0 \sim 0.6)$, which shows obvious degradation of $\bar{v}$ with the increase of HRDD. In this range, the magnitude of flow input has a significant impact on the change of $\bar{v}$. The absolute value of slope of $\bar{v}$ is $32.88 \sim 45.52$ under the peak-period flow input (1000-2000), whereas $26.27 \sim 31.37$ is under the off-peak-period flow input (400-900). The absolute value of slope of $\bar{v}$ is 7.89 20.19 under the high-level range of HRDD (0.6 1). The speed $\bar{v}$ is still sensitive (absolute slope 18.48 20.19) to HRDD under off-peak period flow. However, the speed of near-saturation flow is less sensitive to HRDD, and thus, the speed sensitivity falls (absolute slope 7.89 18.66) under peak-period flow. On the whole, the sensitivity of overall speed to HRDD could be ordered as follows: slight PD and peak flow; slight PD and off-peak flow; heavy PD and off-peak flow; heavy PD and peak flow.

5.6.2. Flow on the Downstream Section. To analyze the impact of flow input and HRDD on the flow on the downstream section $(F)$, flow input is taken as the $x$-axis, HRDD is taken as the $y$-axis, and $F$ is taken as the $z$-axis. The relationship among $F$, flow input, and HRDD is plotted in Figure 10.

The relationship between $F$ and HRDD is as follows. The HRDD change has no significant impact on $F$ when flow input is in a low-level range (400 800). $F$ decreases with the increase of HRDD, when flow input is in a high-level range (800 2000). The larger the flow input is, the more sensitivity of $F$ to HRDD is in this range. The relationship between $F$ and flow input is as follows: $F$ is proportional to flow input in the low-level range of HRDD (0 0.6). The maximum of $F$ does not correspond to flow input $=2000 \mathrm{veh} / \mathrm{h}$ in the highlevel range of HRDD (0.6 1).

There is a phenomenon that $F$ is less than flow input when HRDD is high. From a microscopic perspective, we can explain it as follows. The PD causes the vehicle to decelerate. When flow input is small, even if the vehicle has decelerated, all the vehicles entering the segment can pass per simulation time. As the flow input increases, vehicle queue appears. More seriously, the queue could reach up to the section $x=0 \mathrm{~m}$ on segment. An overflow phenomenon has occurred in the simulation, and some vehicles will be queued outside the research segment. This is the reason why $F<$ flow input in the condition of high HRDD. 


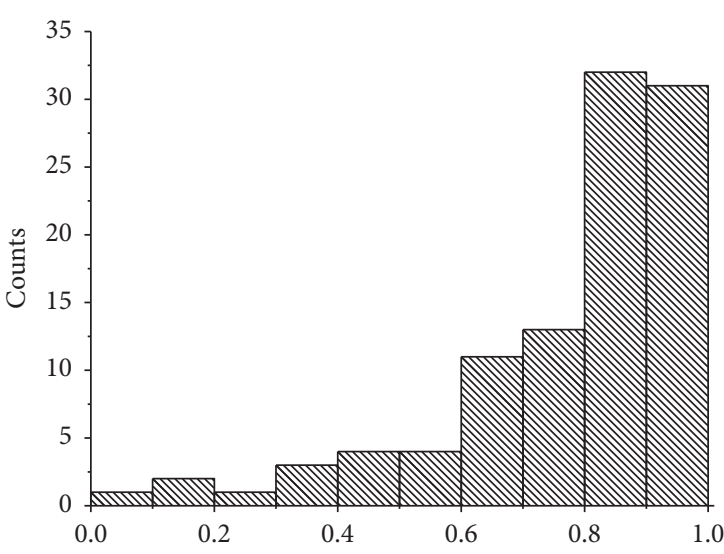

(a)

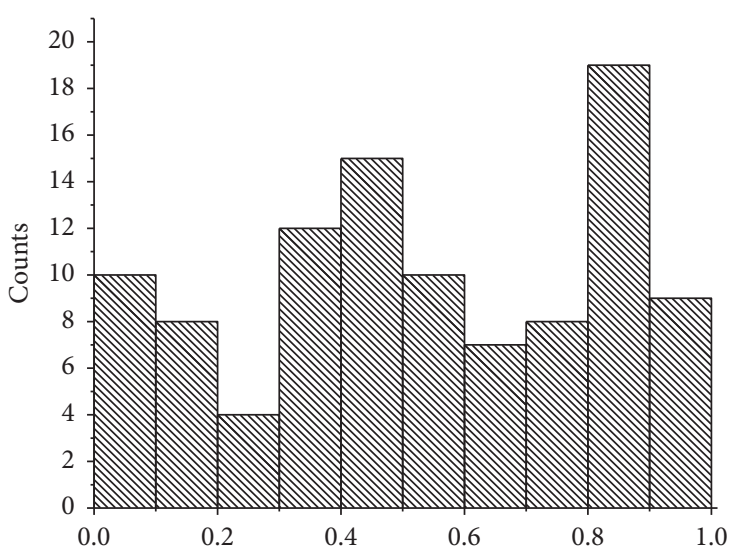

(c)

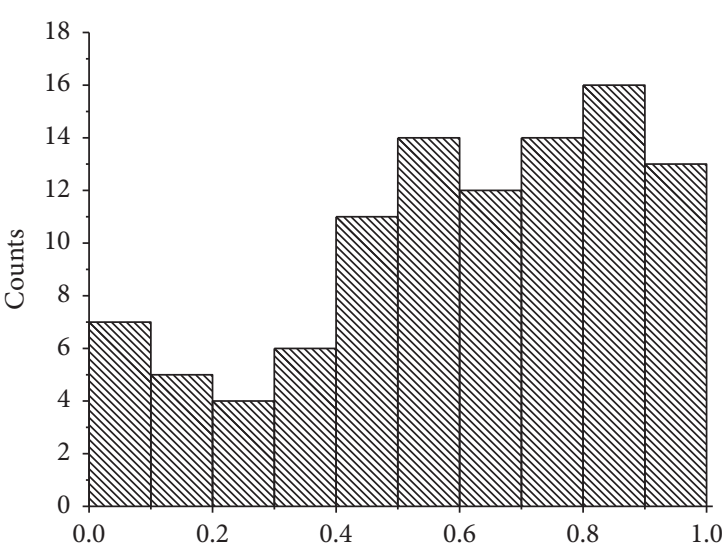

(b)

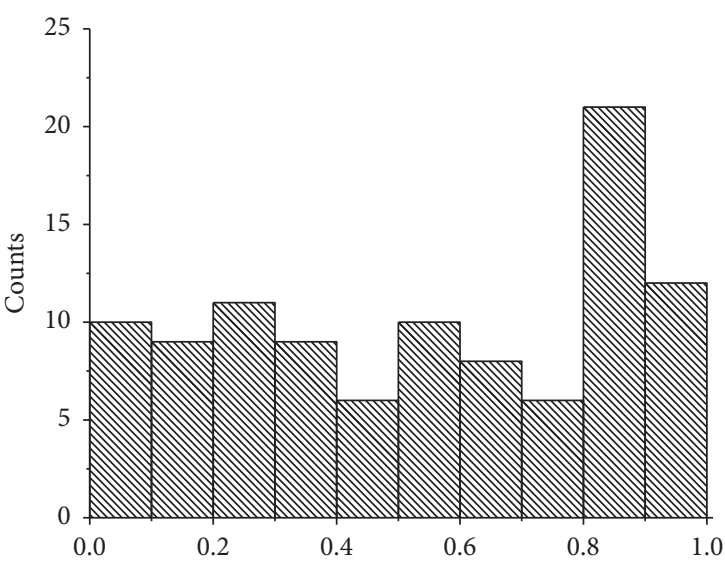

(d)

Figure 8: Distribution rule of each coefficient of simulation scenarios: (a) $D_{0}$; (b) $f_{1}$; (c) $f_{2}$; (d) $f_{3}$.

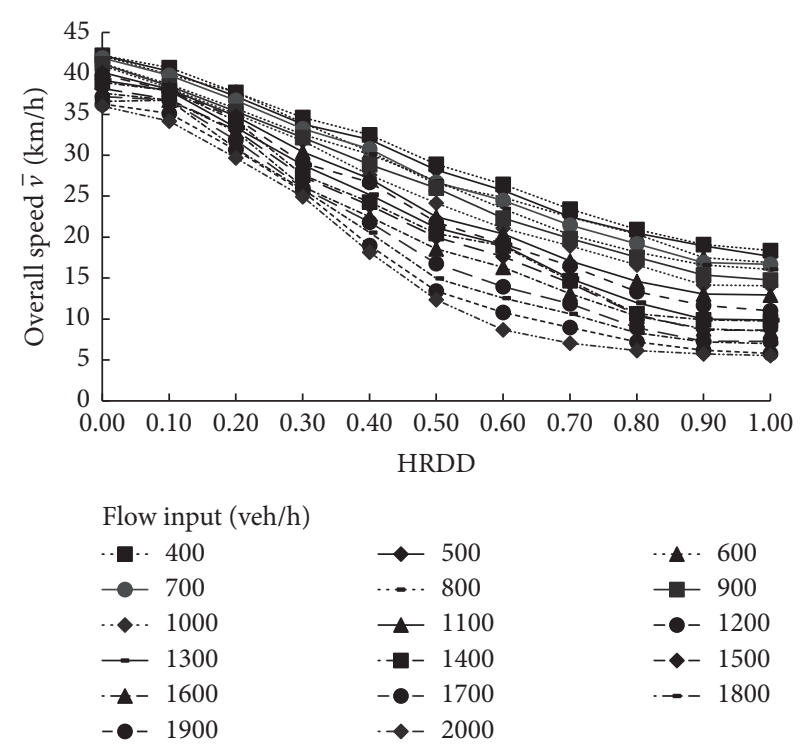

FIgURE 9: Relationship among $\bar{v}$, HRDD, and flow input.

5.6.3. Traffic Capacity. Since we use the design flow of good road as one of the scenarios of flow input, under certain HRDD, the maximum of $F$ out of all the simulation experiments is taken as its road capacity $C$. The relationship

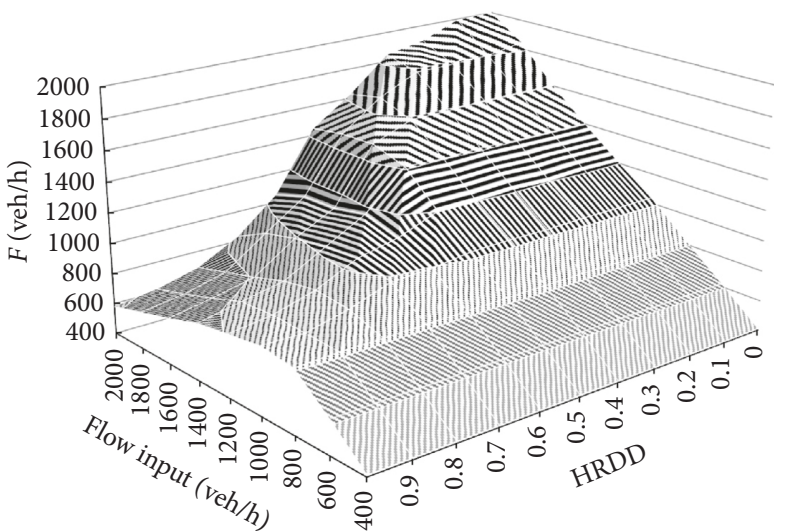

$$
\begin{aligned}
& \text { N 1800-2000 ||| 1000-1200 } \\
& \text { \% 1600-1800 } \\
& \text { N } 1400-1600 \text { N } 600-800 \\
& \equiv 1200-1400 \quad \text { \% } 400-600
\end{aligned}
$$

FIgURE 10: Relationship among F, flow input, and HRDD.

between $C$ and HRDD is plotted with scatter, as shown in Figure 11. When HRDD is in the low-level range (0 0.3), $C$ is stably above $1800 \mathrm{pcu} / \mathrm{h}$. When HRDD is in the moderatelevel range $(0.3 \sim 0.8), C$ varied sharply and declines by up to $40 \%$. When HRDD is in the high-level range (0.8 1), the 


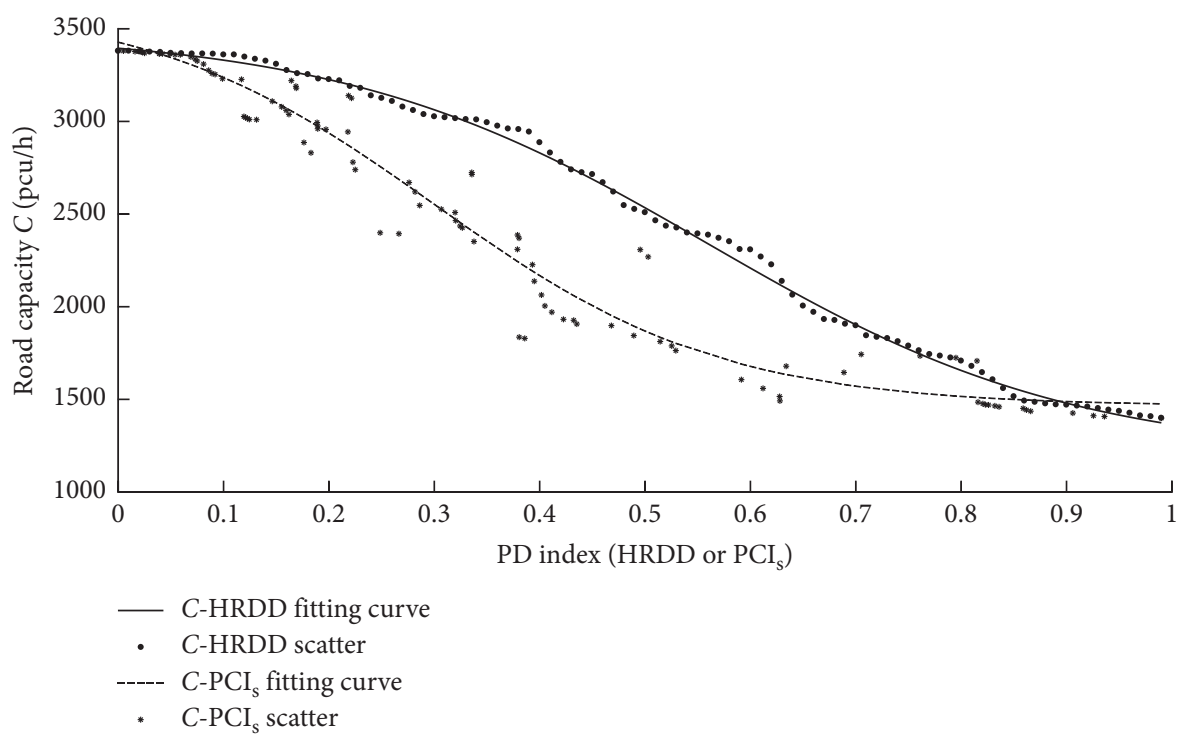

FIGURE 11: Relationship between road capacity and different types of PD indexes.

magnitude of the declines is minor. The DoseResp model is used to fit the scatters. The fitting result is as shown in equation (12). Reduced chi-square and adjust $R$-square are 1251.52 and 0.997, respectively, which meet the requirement of fitting accuracy. The fitting curve shows that the relationship between $C$ and HRDD is roughly in an inverse $S$ shape:

$$
C=1194.31+\frac{2289.23}{\left.1+10^{-2.49(0.56-H R D D}\right)} \text {. }
$$

At present, the PCI index is most frequently used to evaluate the magnitude of road PD. The PCI of the aforementioned 100 scenarios are turned to $\mathrm{PCI}_{\mathrm{s}}$ by normalization processing. The smaller the $\mathrm{PCI}_{\mathrm{s}}$ are, the better the road condition is. The scatters of $C$ corresponding to $\mathrm{PCI}_{S}$ are plotted as shown in Figure 11. The functional relationship between $C$ and $\mathrm{PCI}_{\mathrm{s}}$ is fitted as equation (13). The reduced chi-square and adjust $R$-square are 19814 and 0.87 , respectively, showing that the fitting results meet the accuracy requirements:

$$
C=1462.39+\frac{2180.75}{1+10^{-3.2\left(0.3-\text { PCI }_{s}\right)}}
$$

We find some differences by comparing the two types of fitting curves. $C$ decreases rapidly in the low-level range of $\mathrm{PCI}_{\mathrm{s}}(0 \sim 0.5)$ and then maintains at a stable level in the highlevel range of $\mathrm{PCI}_{\mathrm{s}}(0.5 \sim 1)$. However, $C$ keeps the trend of declining at a relatively constant rate corresponding to HRDD. When HRDD is between 0.1 and 0.8 , the distance difference between the two curves is enlarged.

In addition, the corresponding relationship between HRDD and $\mathrm{PCI}_{\mathrm{s}}$ is as shown in Figure 12. When HRDD is in the range of $(0.1 \sim 0.8)$, the $\mathrm{PCI}_{\mathrm{s}}$ of the same experiment background is generally lower than HRDD. That is the reason why $\mathrm{PCI}_{\mathrm{s}}$ is lower than HRDD corresponding to the same $C$ in Figure 11.

The above differences between two indexes are caused by different calculation methods. While calculating HRDD, the

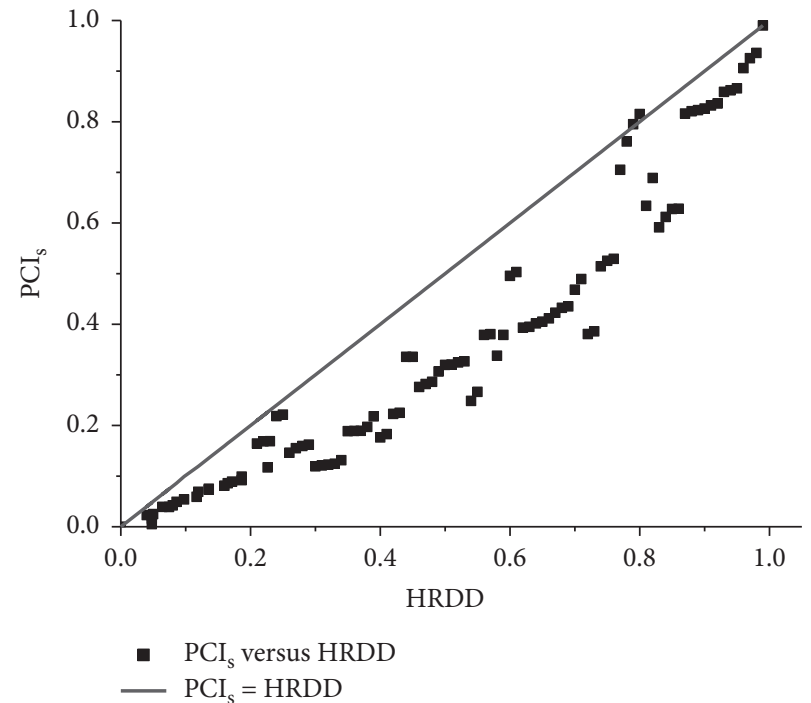

FIGURE 12: Corresponding relationship between HRDD and $\mathrm{PCI}_{\mathrm{s}}$.

initial physical PD degree is discounted terminally because the factors considered such as the sparsely agglomerated PD point layout and the standardized driving behaviors could improve traffic efficiency. As a comparison, $\mathrm{PCI}_{\mathrm{s}}$ only considers the physical structure of the road. Thus, the value of HRDD is smaller in most scenarios.

The proposed index HRDD performs better on the aspect of reflecting traffic efficiency when compared to $\mathrm{PCI}_{\mathrm{s}}$. We can see that HRDD has a relatively stable sensitivity to $C$ in the whole range of the HRDD value, whereas $\mathrm{PCI}_{\mathrm{s}}$ cannot reflect the changing of traffic efficiency in part range $(0.5 \sim 1)$ in Figure 11.

\section{Conclusion}

This paper divides the road into lane cells and then defines the PD degree of lane cell by calculating the reduction ratio 
of average speed. In the next step, four coefficients, namely, (1) the aggregated PD value of road, (2) the density coefficient of PD cells, (3) the dispersion coefficient of PD cells, and (4) the lane-changing coefficient, are proposed to describe four aspects of the impact on the traffic efficiency. HRDD is synthesized by these four coefficients. The feasibility of the calculation process and performance of HRDD are analyzed through the case study.

The result shows that the inverse correlation of the overall speed with flow rate and HRDD. The sensitivity of overall speed to HRDD could be ordered as following intervals: slight PD and peak flow; slight PD and off-peak flow; heavy PD and off-peak flow; heavy PD and peak flow. The increase of flow rate would aggravate the traffic efficiency loss under the same PD scenario. The road capacity and $\mathrm{HRDD}$ are in inverse relationship. Compared to $\mathrm{PCI}_{\mathrm{s}}$ calculated only based on physical structure features of PD points, the value of HRDD is smaller in the same PD scenario. Additionally, HRDD has a relatively stable sensitivity to road capacity in the whole range of the HRDD value, whereas $\mathrm{PCI}_{s}$ cannot reflect the changing of road capacity in part range (0.5 1). It indicates that HRDD is more suitable to illustrate the variation of traffic efficiency under the PD.

Most previous PD indicators are only applicable to civil engineering, whereas there is a wider scope for the application of HRDD. Our proposed index could be used to guide the management of transportation infrastructures and travel demand.

\section{Data Availability}

The data used to support the findings of this study are available from the corresponding author upon request.

\section{Conflicts of Interest}

The authors declare that there are no conflicts of interest regarding the publication of this paper.

\section{Authors' Contributions}

Shun Guo contributed to the formal analysis, investigation, and writing of the original draft. Zhengfeng Huang contributed significantly to the conceptualization and methodology. Pengjun Zheng as the project administrator provided the funding. Ting Lu contributed to the simulation. Lili Lu helped in the model validation. Xuan Li was responsible for review and editing.

\section{Acknowledgments}

This research was funded by the Zhejiang Provincial Natural Science Foundation of China (grant number LY18E080009), National Natural Science Foundation of China (grant numbers 51408321 and 71701108), Zhejiang Philosophy and Social Science Program (grant numbers 16NDJC015Z and 18NDJC015Z), and Natural Science Foundation of Ningbo (grant number 2017A610139).

\section{References}

[1] C. Deyoung, "Development of concepts for pavement management," American Journal of Mental Deficiency, vol. 70, no. 3, pp. 443-447, 1979.

[2] M. Y. Shahin and S. D. Kohn, "Development of a Pavement Condition Rating Procedure for Roads, Streets, and Parking Lots," Conditions Rating Procedure, Vol. 1, Construction Engineering Research Lab (Army), Champaign, IL, USA, 1979.

[3] T. Sosuke and K. Satoshi, "Measurement method of crack rate in road pavement maintenance management using smartphone," Graduate School of Engineering, Nagasaki University, vol. 48, no. 91, pp. 22-30, 2018.

[4] S. Mcrobbie, L. Walter, C. Read et al., "Developing SCANNER road condition indicator parameter thresholds and weightings-version 1," TRL Published Project Report, The National Academies of Sciences, Engineering, and Medicine, Washington, DC, USA, 2007.

[5] China Communication Press, Highway Performance Assessment Standards, Vol. 17, China Communication Press, Beijing, China, 2018.

[6] S. A. Arhin and E. C. Noel, "Predicting pavement condition index from international roughness index in Washington, DC," No. DDOT-DT-03, The National Academies of Sciences, Engineering, and Medicine, Washington, DC, USA, 2014.

[7] S. Chandra, "Effect of road roughness on capacity of two-lane roads," Journal of Transportation Engineering, vol. 130, no. 3, pp. 360-364, 2004.

[8] J. Ben-Edigbe and N. Ferguson, "Extent of capacity loss resulting from pavement distress," Proceedings of the Institution of Civil Engineers-Transport, vol. 158, no. 1, pp. 27-32, 2005.

[9] A. O. Daniel, D. S. Matawal, F. Aitsebaomo, and E. B. Ojo, "The extent of travel time increment due to pavement distress," Journal of Engineering \& Applied Sciences, vol. 9, no. 10, pp. 2016-2021, 2014.

[10] J. Ben-Edigbe, N. Mashros, and A. Minhans, "Exploration of trapezoidal flowrate contractions resulting from pavement distress," Journal of Emerging Trends in Engineering \& Applied Sciences, vol. 2, no. 2, pp. 351-354, 2011.

[11] R. Bridgelall, J. Hough, and D. Tolliver, "Characterising pavement roughness at non-uniform speeds using connected vehicles," International Journal of Pavement Engineering, vol. 2, pp. 1-7, 2017.

[12] W. Zheng, D. Hu, J. Wang, and C. Wang, "Fault localization analysis based on deep neural network," Mathematical Problems in Engineering, vol. 2016, Article ID 1820454, 11 pages, 2016.

[13] E. I. B. J. Schroeder and N. M. Rouphail, "A framework for evaluating pedestrian-vehicle interactions at unsignalized crossing facilities in a microscopic modeling environment," in Proceedings of the Transportation Research Board 86th Annual Meeting, Washington, DC, USA, January 2007.

[14] R. Z. Koshy and V. T. Arasan, "Influence of bus stops on flow characteristics of mixed traffic," Journal of Transportation Engineering, vol. 131, no. 8, pp. 640-643, 2005.

[15] D. R. R. Reddy, "Evaluation of rate of the reduction in road capacity at kerb side bus stop in urban areas under mixed traffic conditions-a case study in Hyderabad city," IOSR Journal of Mechanical and Civil Engineering, vol. 14, no. 4, pp. 1-8, 2017. 
[16] T. A. Domencich and G. Kraft, "Urban travel demand: a behavioral analysis," Transportation Research, vol. 10, no. 4, pp. 128-130, 1975.

[17] X. Zhao, Y. P. Wu, G. Ren, K. Ji, and W. W. Qian, "Clustering analysis on ridership patterns at subway stations: a case in Nanjing, China," Journal of Urban Planning and Development, vol. 145, no. 2, article 04019005, 2019. 


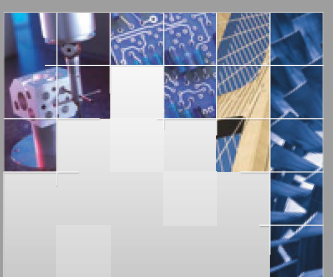

\section{Enfincering}
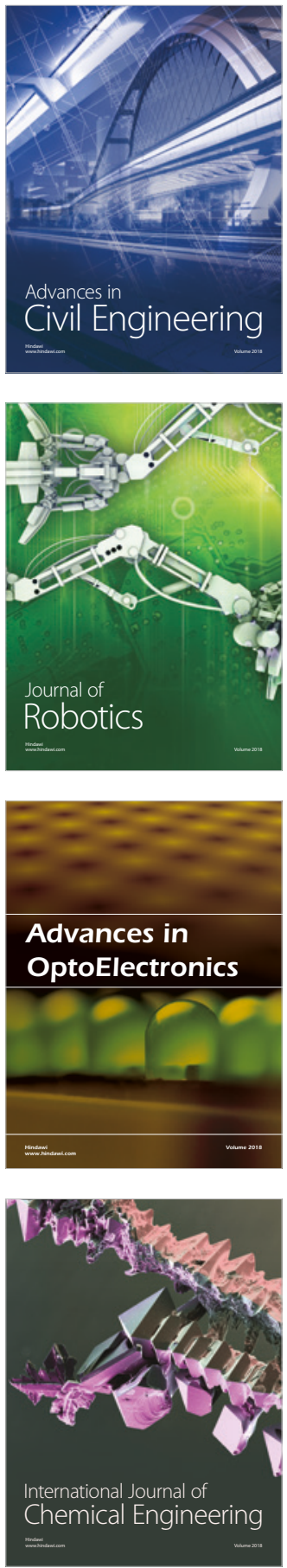

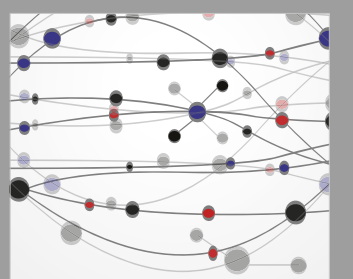

\section{Rotating \\ Machinery}

The Scientific World Journal

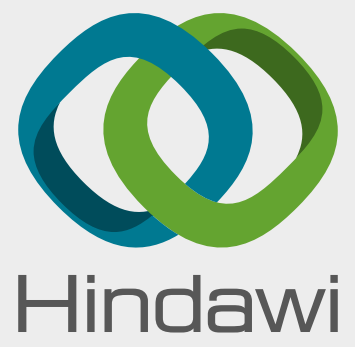

Submit your manuscripts at

www.hindawi.com
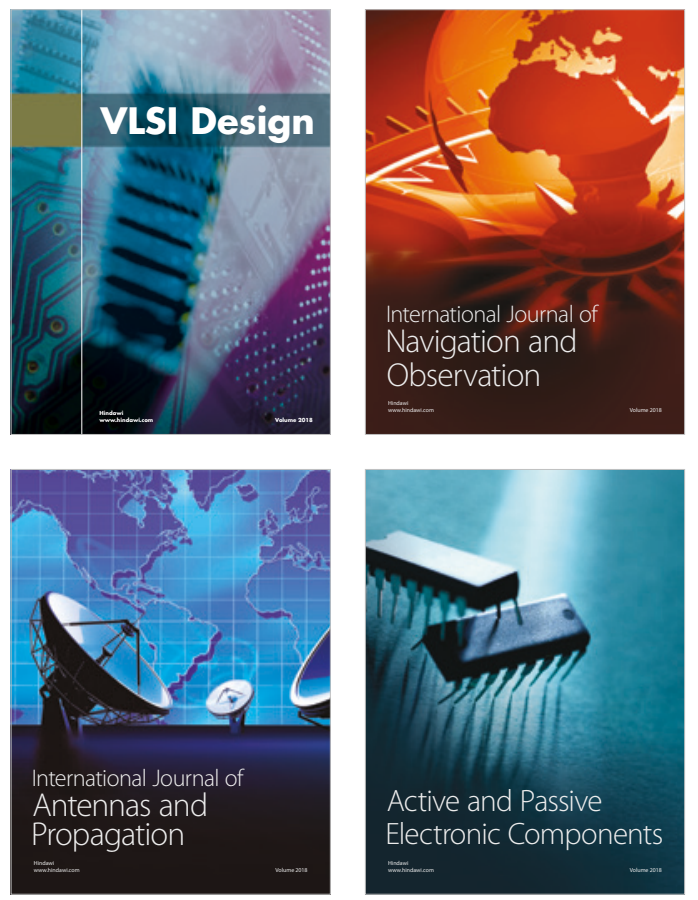
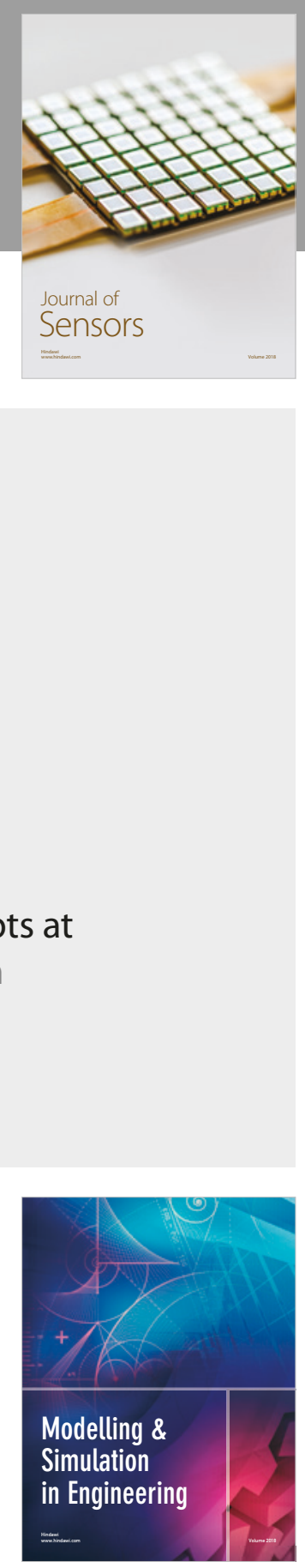

\section{Advances \\ Multimedia}
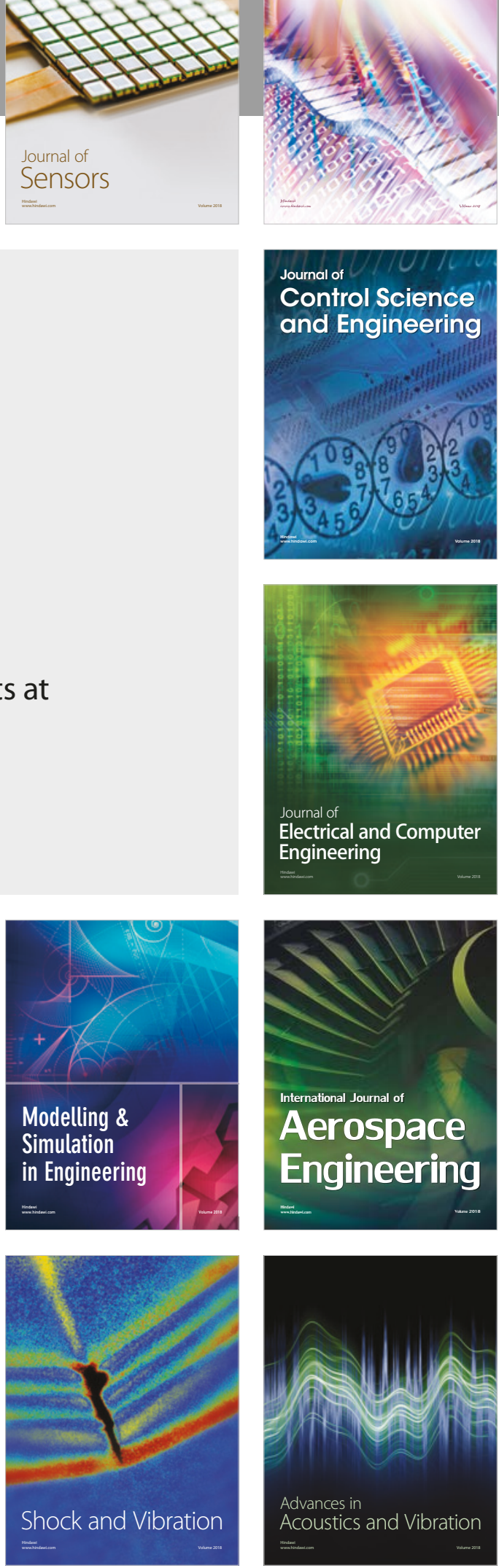